\title{
THE DISTINGUISHED BOUNDARY OF THE UNIT OPERATOR BALL
}

\author{
EDWARD NELSON
}

TheOREM. Let $B$ be the set of all operators $A$ on a Hilbert space $H$ with $\|A\| \leqq 1$, and let $\mathcal{u}$ be the set of unitary operators on $H$. If $f$ is a strongly continuous complex valued function on $\mathbb{B}$, such that its restriction to the intersection of $B$ with any finite dimensional linear subspace of operators is holomorphic, then

$$
|f(A)| \leqq \sup _{U \in \mathcal{U} .}|f(U)|
$$

for all $A$ in $B$.

Proof. Consider operators of the form $A \oplus 1_{N \perp}$ where $N$ is a finite dimensional subspace of $H, A$ is an operator on $N$ with $\|A\| \leqq 1$, and $1_{N \perp}$ is the identity on $N^{\perp}$. Such operators are clearly dense in $B$ in the strong operator topology, and $U \oplus 1_{N \perp}$ is unitary if $U$ is unitary on $N$. Therefore it is enough to prove (1) for finite dimensional spaces. In that case (1) follows from work of Bochner [1], or alternatively it may be proved as follows. If $A$ is an $n \times n$ matrix with $\|A\| \leqq 1$ we may write $A$ in polar form as $A=V P$ where $V$ is unitary and $P$ is diagonal with entries $\lambda_{i}, 0 \leqq \lambda_{i} \leqq 1$. Consider the set of all matrices $V Q$ where $Q$ is diagonal with entries $\zeta_{i},\left|\zeta_{i}\right| \leqq 1$. For fixed $i$, and for fixed values of $\zeta_{j}(j \neq i), f(V Q)$ is a holomorphic function of $\zeta_{i}$. Apply the maximum modulus theorem $n$ times to get $|f(A)|$ $\leqq|f(V Q)|$, where $Q$ is diagonal with entries $\zeta_{i}$ on the unit circle, and thus conclude that $U=V Q$ is unitary.

As a particular case, let $p$ be a polynomial in one variable with complex coefficients, $p(z)=c_{0}+c_{1} z+\cdots+c_{k} z^{k}$, such that

$$
\sup _{|z| \leqq 1}|p(z)| \leqq 1 \text {. }
$$

Define $p(A)=c_{0}+c_{1} A+\cdots+c_{k} A^{k}$. Then for any vectors $u$ and $v$ in $H, f(A)=(p(A) u, v)$ satisfies the hypotheses of the theorem. For unit vectors $u$ and $v$, (1) yields

$$
|(p(A) u, v)| \leqq \sup _{\boldsymbol{U} \in \mathcal{U} .}|(p(U) u, v)| \leqq \sup _{U \in \mathcal{U}}\|p(U)\|,
$$

and since this holds for all unit vectors $u$ and $v$ we conclude that $\|p(A)\| \leqq \sup _{U \in \mathcal{U}}\|p(U)\|$. But by the spectral theorem for unitary

Received by the editors June 26, 1961. 
operators (or $n \times n$ matrices, by approximation), $\|p(U)\| \leqq 1$, so that $\|p(A)\| \leqq 1$ for all $A$ in $B$. By continuity, this holds for any function $p$ continuous in the closed unit disc, holomorphic in its interior, and satisfying (2). This was first proved by von Neumann [3] in 1951 (see also [2]).

\section{REFERENCES}

1. S. Bochner, Group invariance of Cauchy's formula in several variables, Ann. of Math. vol. 45 (1944) pp. 686-707.

2. E. Heinz, Ein v. Neumannscher Satz über beschränkte Operatoren im Hilbertschen Raum, Nachr. Akad. Wiss. Göttingen. Math.-Phys. Kl. IIa. (1952) pp. 5-6.

3. J. von Neumann, Eine Spektraltheorie für allgemeine Operatoren eines unitären Raumes, Math. Nachr. vol. 4 (1951) pp. 258-281.

Princeton University

\section{A PROOF OF LIOUVILLE'S THEOREM}

\section{EDWARD NELSON}

Consider a bounded harmonic function on Euclidean space. Since it is harmonic, its value at any point is its average over any sphere, and hence over any ball, with the point as center. Given two points, choose two balls with the given points as centers and of equal radius. If the radius is large enough, the two balls will coincide except for an arbitrarily small proportion of their volume. Since the function is bounded, the averages of it over the two balls are arbitrarily close, and so the function assumes the same value at any two points. Thus a bounded harmonic function on Euclidean space is a constant.

\section{Princeton University}

Received by the editors June 26, 1961. 